se Regeln dienen nicht nur dazu, gewisse Grenzen zu setzen, sondern sollen das »lebenspraktische Training « unterstützen. Dieses wiederum soll den Jugendlichen helfen vor allem im Berufsleben zurechtzukommen, denn häufig scheitert eine angefangene Ausbildung daran, dass der Jugendliche regelmäßig zu spät kommt oder sich den dortigen Bedingungen nicht anpassen kann. Eine 24-Stunden-Bereitschaft seitens der Betreuer ist nicht gegeben, diese sind im Notfall aber über Handy zu erreichen.

Die Ziele des Vereins sind neben der Lebensführung ohne Straftaten, die Entwicklung größtmöglicher Selbstbestimmung und die Entwicklung sozialer Verantwortung.

Der Hauptunterschied zum Strafvollzug ist, dass die Jugendlichen das neu erlernte Wissen und Können direkt im Alltagsleben ausprobieren können. Erfolgserlebnisse wirken dabei als positive Verstärker und neu erworbene Verhaltensmuster manifestieren sich. Die erlernten Verhaltensmuster führen dazu, dass sie sich besser in das Gesellschaftsleben integrieren können. Rückfälle in alte Verhaltensmuster werden von den Betreuern erkannt und mit den Betroffenen in Einzelgesprächen thematisiert. In einigen Jugendstrafanstalten werden zwar auch Trainingsprogramme unterschiedlichster Art angeboten z.B. Anti-Aggressivitätstraining. Aber hier ist der Weg des Trainings bis zur konkreten Alltagssituation zu lang: Das Erlernte hat sich nicht genügend verfestigt, um sofort im Alltagsleben zu funktionieren. Dies führt nach der Entlassung nicht nur häufig zu Frustration und den Rückfall in alte Verhaltensmuster, sondern auch dazu, das möglicherweise eine erneute Straftat begangen wird.

Das Konzept der Jugendwohngemeinschaft geht jedoch nur auf, wenn der Jugendliche vor, während oder spätestens nach seinem Aufenthalt dort, erkennt, dass er sich selbst und seine Verhaltensweisen ändern muss, um in Zukunft straffrei zu bleiben. Fehlt ihm diese Einsichtsfähigkeit, so wird er den Kreislauf der kriminellen Karriere nicht durchbrechen können. Diese Erfahrung musste auch der Verein Maßstab e.V. machen. Langzeitstudien gibt es bisher nicht, während des Aufenthaltes blieben jedoch $80 \%$ der Jugendlichen straffrei. Diese Quote ergibt sich aus der
Evaluationsliste der Jugendwohngemeinschaft, die seit 1999 geführt wird. Der Zeitraum dieser Liste erstreckt sich jeweils über ein Kalenderjahr. In dieser Liste werden neben den Daten der Jugendlichen folgende Spalten für jeden einzelnen Bewohner geführt und bei Vorliegen entsprechend eingetragen: Verweildauer in Monaten insgesamt/reguläre Beendigung/JA stellt ein/Abbruch wg. Regelverstoß/Abbruch wg. erneuter Straffälligkeit während des Aufenthaltes/Abbruch auf Wunsch des Bewohners/Abbruch aus anderen Gründen/straftatenfreies Leben während des Aufenthaltes/erneute Straffälligkeit innerhalb von 6 Monaten nach Entlassung/erneute Straffälligkeit innerhalb von 12 Monaten nach Entlassung/Bewohner hat schulische und berufliche Orientierung gewonnen/Bewohner ist ein Ausbildungsverhältnis eingegangen/Abbruch eines Ausbildungsverhältnisses nach 1-3 Monaten/Abbruch eines Ausbildungsverhältnisses nach mehr als 3 Monaten sowie Ausbildungsverhältnis bestand bei Beendigung fort.

Nicht nur die im Gegensatz zu den Statistiken der Jugendstrafanstalten positive Bilanz ist ein Argument für das Projekt, sondern auch die Kosten: Die Kosten der Unterbringung betragen $120 €$ pro Tag und Person. Die Unterbringung eines Jugendlichen im Jugendstrafvollzug bzw. in der Untersuchungshaft liegen ca. 20-30 € über diesem Tagessatz. Die Jugendlichen, die während der Unterbringung in der Wohngemeinschaft erwerbstätig sind, haben an die Kostenträger einen Eigenanteil von bis zu 70\% zu leisten. Durch das Projekt Haftvermeidung ergeben sich jährlich hohe Einsparungen für das Land NRW.

Da in einem solchen Projekt die negativen Auswirkungen der traditionellen Jugendstrafe vermieden werden, ohne dass sich die Rückfallquote erhöht, ist diesem unbedingter Vorzug zu geben.

Die Verfasserinn ist Sozialarbeiterin in Köln

\section{Fußnoten:}

1 Die Ausführungen betreffen nur den Verein Maßstab e.V., da nur dieser von der Verfasserin ausführlich untersucht worden ist und keine anderen Einrichtungen ähnlicher Art.

$2 \mathrm{Im}$ folgenden Text wird aufgrund der besseren Lesbarkeit nur noch von dem Jugendlichen gesprochen.

TERMINAL

\title{
Europäisches Parlament wehrt sich gegen Fluggastdaten-Transfer an US-Sicherheitsbehörden
}

\author{
Von Rolf Gössner
}

Das Europäische Parlament wird den Europäischen Gerichtshof in Luxemburg anrufen, um die Annullierung eines Abkommens zu erreichen, das EU-Rat und -Kommission im Mai 2004 gegen das ausdrückliche Votum des Parlaments und der EUDatenschutzbeauftragten mit den USA geschlossen hatten. In dem Abkommen geht es um die Übermittlung von Flugpassagierdaten an Sicherheitsbehörden der Vereinigten Staaten.

Von dem Datentransfer sind jährlich über zehn Millionen Flugpassagiere aus Europa, die in die USA fliegen oder über die USA weiterreisen, unmittelbar betroffen. Schon bevor sie auch nur einen Fuß auf den Boden des Landes gesetzt haben, sind die US-Sicherheitsbehörden über sie umfassend informiert, haben ihre Schlüsse aus den zum Teil hochsensiblen Informationen gezogen, Verdachtsmomente ventiliert oder Verdächtigungen konstruiert. Die gelieferten Daten können nach allen Richtungen verarbeitet, durchgerastert und mit einer Unzahl anderer polizeilicher, geheimdienstlicher oder auch privater Dateien abgeglichen werden, um Bewegungsbilder und Persönlichkeitsprofile $\mathrm{zu}$ erstellen und Verdächtige herauszufiltern.

Auch unbescholtene Fluggäste müssen verstärkt damit rechnen, dass sie auf diese Weise zu Opfern rigider Antiterror-Maßnahmen werden und sich wie Verbrecher behandeln lassen müssen. Die Datenübermittlung kann letztlich zu peinlichen Verhören und erkennungsdienstlicher Behandlung führen, $\mathrm{zu}$ willkürlichen Festnahmen und Inhaftierungen und schließlich zu Ausweisungen auch vollkommen unschuldiger Personen. Beispiele hierfür gibt es schon genug. Begründet wird all dies mit der »Bekämpfung des Terrorismus sowie sonstiger Verbrechen transnationaler Art, einschließlich der organisierten Kriminalität«.

Doch die große Mehrheit der Europa-Parlamentarier vermisst einen ausreichenden Schutz für die per Online-Zugriff übermittelten Daten. Die erzwungene Datenübermittlung verstoße gegen Grundrechte und völkerrechtlich garantierte Prinzipien des europäischen Datenschutzes, zumal die Daten auch an Behörden anderer Staaten weitergegeben werden dürfen. Datenschützer halten den Datentransfer, der Fluggäste aus der EU zu gläsernen Passagieren macht, für eine unverhältnismäßige und ungeeignete Maßnahme, die weit über das Ziel der Terrorbekämpfung hinausschieße und sich auch mit dem anerkennenswerten Sicherheitsinteresse nicht rechtfertigen lasse.

Dr. Rolf Gössner, Rechtsanwalt und Publizist, ist Präsident der "Internationalen Liga für Menschenrechte", die eine gerichtliche Überprüfung des DatentransferAbkommens angemahnt hat. 